\title{
Simultaneous Determination of Cysteamine and Cystamine in Cosmetics by lon-Pairing Reversed-Phase High-Performance Liquid Chromatography
}

\author{
Yejin Kim and Dong Hee Na \\ College of Pharmacy, Chung-Ang University, Seoul, Korea
}

\begin{abstract}
Cysteamine has been used in cosmetics as an antioxidant, a hair straightening agent, and a hair waving agent. However, recent studies indicate that cysteamine can act as an allergen to hairdressers. The objective of this study was to develop and validate a simple and effective reversed-phase high-performance liquid chromatography (RPHPLC) method for the measurement of cysteamine and its dimer, cystamine. Sodium 1-heptanesulfonate (NaHpSO) was used as an ion-pairing agent to improve chromatographic performance. Separation was performed on a Gemini C18 column $(250 \mathrm{~mm} \times 4.6 \mathrm{~mm}, 5 \mu \mathrm{m}$ particle size) using a mobile phase composed of 85:15(v/v) $4 \mathrm{mM}$ $\mathrm{NaHpSO}$ in $0.1 \%$ phosphoric acid:acetonitrile. UV absorbance was monitored at $215 \mathrm{~nm}$. The RP-HPLC method developed in this study was validated for specificity, linearity, limit of detection, limit of quantitation, precision, accuracy, and recovery. Cysteamine and cystamine were chromatographically resolved from other reducing agents such as thioglycolic acid and cysteine. Extraction using water and chloroform resulted in the recovery for cysteamine and cystamine ranging from $100.2-102.7 \%$ and $90.6-98.7 \%$, respectively. This validated RP-HPLC method would be useful for quality control and monitoring of cysteamine and cystamine in cosmetics.
\end{abstract}

Key words: Cysteamine, Cystamine, Cosmetics, Reversed-phase high-performance liquid chromatography, Ionpairing agent

\section{INTRODUCTION}

Cysteamine is an aminothiol compound (HS- $\mathrm{CH}_{2}-\mathrm{CH}_{2}-$ $\mathrm{NH}_{2}$ ) endogenously formed by degradation of coenzyme A and it can be converted to the neurotransmitter hypotaurine by cysteamine dioxygenase (1). Cysteamine has been used as a therapeutic agent for the treatment of cystinosis, cystic fibrosis, neurodegenerative disorders such as Huntington's disease and Parkinson's disease, and nonalcoholic fatty liver disease $(2,3)$. In 1994, cysteamine was first approved as a drug product for the treatment of cystinosis, which is characterized by accumulation of cystine

Correspondence to: Dong Hee $\mathrm{Na}$, College of Pharmacy, ChungAng University, 84 Heukseok-ro, Dongjak-gu, Seoul 06974, Korea E-mail:dhna@cau.ac.kr

This is an Open-Access article distributed under the terms of the Creative Commons Attribution Non-Commercial License (http:// creativecommons.org/licenses/by-nc/3.0) which permits unrestricted non-commercial use, distribution, and reproduction in any medium, provided the original work is properly cited. in cells throughout the body (4).

Cysteamine hydrochloride is also used in cosmetics as an antioxidant, a hair straightening agent, and a hair waving agent. It functions as a reducing agent at concentrations between $5 \%$ and $12 \%$ in permanent-wave solutions (5). However, recent studies in Europe and Japan reported that cysteamine can act as an allergen to hairdressers (68). Therefore, cysteamine needs to be evaluated for its potential as an allergen due to its presence in hair waving and dyeing agents (8).

Cysteamine has been analyzed by several analytical techniques, including high-performance liquid chromatography (HPLC) with electrochemical detection (9), fluorescence detection $(10,11)$ and ultraviolet-absorbance detection (12), capillary electrophoresis (13), and gas chromatography with flame photometric detection $(14,15)$. However, most of these methods were developed for the analysis of biological fluid samples with complicated sample pretreatments, such as derivatization, and no studies have evaluated cysteamine in cosmetics. 
The purpose of this study was to develop a simple and effective analytical method for the quantitation of cysteamine using reversed-phase high-performance liquid chromatography (RP-HPLC). As cysteamine can be readily oxidized to the disulfide form cystamine $\left(\mathrm{NH}_{2}-\mathrm{CH}_{2}-\right.$ $\mathrm{CH}_{2}-\mathrm{S}-\mathrm{S}-\mathrm{CH}_{2}-\mathrm{CH}_{2}-\mathrm{NH}_{2}$ ), a RP-HPLC method for simultaneous determination of cysteamine and cystamine was developed and validated. The method was then used to analyze cysteamine and cystamine in hair cosmetics.

\section{MATERIALS AND METHODS}

Materials. Cysteamine hydrochloride (CAS No. 15657-0), cystamine dihydrochloride (CAS No. 56-17-7), and L-cysteine were obtained from Sigma-Aldrich (St. Louis, MO, USA). Acetonitrile (HPLC grade) was obtained from Fisher Scientific (Fair Lawn, NJ, USA). Sodium 1-heptanesulfonate (NaHpSO) and calcium thioglycolate trihydrate were purchased from TCI (Tokyo, Japan). All other chemicals were of analytical grade or the best grade available. Two commercial cosmetics and creams were purchased from a local store in Korea.

Preparation of standard solutions. Each stock solution of cysteamine or cystamine was prepared at a concentration of $5 \mathrm{mg} / \mathrm{mL}$ in deionized water. Working standard solutions were prepared by diluting of the stock solutions with deionized water to a concentration range of $2-500 \mu \mathrm{g} /$ $\mathrm{mL}$ for cysteamine and $5-500 \mu \mathrm{g} / \mathrm{mL}$ for cystamine. Every working standard solution was immediately prepared and analyzed.

HPLC analysis. All HPLC experiments were performed on a Dionex HPLC system (Dionex, Sunnyvale, CA, USA) equipped with an auto-sampler, pump, column compartment, and 4-channel multi UV-Vis detector. Data were collected and analyzed using Chromeleon 6.8 software (Dionex). Separation was performed using an isocratic elution program on a Gemini C18 column (4.6 mm i.d. $\times$ $250 \mathrm{~mm}, 5 \mu \mathrm{m}$ particle size; Phenomenex, Torrance, CA, USA) with a guard column $(3.0 \mathrm{~mm}$ i.d. $\times 40 \mathrm{~mm}$; Phenomenex). The mobile phase consisted of $85: 15(\mathrm{v} / \mathrm{v})$ aqueous $4.0 \mathrm{mM} \mathrm{NaHpSO}$ in $0.1 \%$ phosphoric acid: acetonitrile. The injection volume was $20 \mu \mathrm{L}$ and the flow rate was $1.0 \mathrm{~mL} / \mathrm{min}$. The column temperature was maintained at $25^{\circ} \mathrm{C}$ and the detection wavelength was set at $215 \mathrm{~nm}$.

Validation of HPLC methods. The validation procedure was conducted in according with ICH guidelines (16). The selectivity of the method was assessed by analyzing mixtures of cysteamine-cystamine, cysteaminethioglycolic acid, or cysteamine-cysteine. Linearity was determined by plotting peak area of standards versus con- centrations of standard and performing least-squares linear regression across the ranges of 2 to $500 \mu \mathrm{g} / \mathrm{mL}$ for cysteamine and 5 to $500 \mu \mathrm{g} / \mathrm{mL}$ for cystamine. Curve fit was determined by the coefficient of determination $\left(r^{2}\right)$. The limit of detection (LOD) and the limit of quantitation (LOQ) were calculated according to the following formulas: $\mathrm{LOD}=3.3(\mathrm{~s} / \mathrm{m})$ and $\mathrm{LOQ}=10(\mathrm{~s} / \mathrm{m})$. Where $\mathrm{s}$ represents the standard deviation of the blank measurements $(\mathrm{n}=7)$ as the minimum detectable level and m represents the slope of the calibration curve. The precision and accuracy of the method were determined by analyzing five replicates for intra-day and three replicates for inter-day analyses. Precision and accuracy were determined using quality control (QC) samples containing cysteamine (2, $15,150$, and $500 \mu \mathrm{g} / \mathrm{mL})$ and cystamine $(5,15,150$, and $500 \mu \mathrm{g} / \mathrm{mL})$. Recovery was evaluated by comparing the peak areas of each $100 \mu \mathrm{g} / \mathrm{mL}$ cysteamine and cystamine spiked into blank cream prior to extraction to the peak areas of $100 \mu \mathrm{g} / \mathrm{mL}$ cysteamine and cystamine spiked into blank cream post-extraction $(17,18)$.

Analysis of cosmetics. Approximately $1.0 \mathrm{~g}$ of the commercial product was weighed into a volumetric flask and then dissolved in $100 \mathrm{~mL}$ of deionized water. The solution was transferred to a separatory funnel and the aqueous phase from the funnel was collected after rinsing twice with $20 \mathrm{~mL}$ of chloroform. The collected aqueous phase was centrifuged at 3,000 rpm for $10 \mathrm{~min}$. The clear supernatant was passed through a $0.45 \mu \mathrm{m}$ PTFE membrane filter and injected onto the HPLC system for quantitative determination of cysteamine and cystamine in cosmetics.

\section{RESULTS}

Analytical method development. Cysteamine and cystamine are hydrophilic molecules that contain amine functional groups, which presented a challenge with regard to retention on a $\mathrm{C} 18$ column with a mobile phase composed of aqueous $0.1 \%$ phosphoric acid and acetonitrile. As shown in Fig. 1A, cysteamine and cystamine had retention times of $2.4 \mathrm{~min}$ and $2.1 \mathrm{~min}$, respectively, when separated using a mobile phase composed of $15 \%$ acetonitrile and $85 \%$ aqueous $0.1 \%$ phosphoric acid. Therefore, we used $\mathrm{NaHpSO}$ as an ion-pairing agent to improve retention based on the cations present in cysteamine and cystamine. Using a mobile phase composition of $85: 15$ aqueous $4.0 \mathrm{mM} \mathrm{NaHpSO}$ with $0.1 \%$ phosphoric acid: acetonitrile, cysteamine and cystamine eluted at $6.4 \mathrm{~min}$ and 15.9 min, respectively, as shown in Fig. 1B. Cysteamine and cystamine in samples were identified by retention time comparison to prepared authentic standards, as shown in Fig. 1C and 1D. To optimize separation of cysteamine and cystamine, mobile phases containing $0,3.5,4.0,4.5$, and $5.0 \mathrm{mM} \mathrm{NaHpSO}$ were evaluated. Use of $3.5 \mathrm{mM}$ 


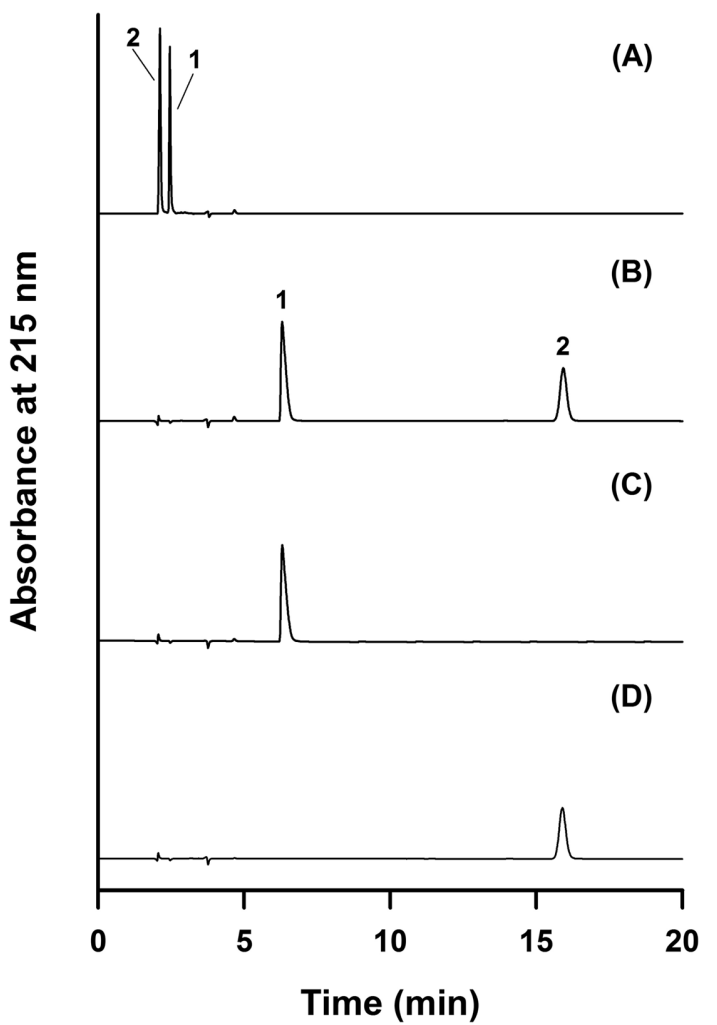

Fig. 1. RP-HPLC analysis of cysteamine and its dimer cystamine. Chromatogram of cysteamine and cystamine obtained with a mobile phase composed of $85: 15(\mathrm{v} / \mathrm{v}) \quad 0.1 \%$ phosphoric acid:acetonitrile without ion-pairing agent $(A)$ and chromatograms of a mixture of cysteamine and cystamine (B), cysteamine only $(C)$, and cystamine only (D) obtained with a mobile phase composed of 85:15 (v/v) $4.0 \mathrm{mM} \mathrm{NaHpSO}$ as ion-pairing agent in $0.1 \%$ phosphoric acid:acetonitrile. Peak 1 : cysteamine and peak 2: cystamine.

$\mathrm{NaHpSO}$ resulted in a broad cystamine peak. At $4.0 \mathrm{mM}$ $\mathrm{NaHpSO}$ and higher, cysteamine and cystamine were retained well, and their peaks exhibited good symmetry. Thus, the optimal concentration of NaHpSO for separation of cysteamine and cystamine was $4.0 \mathrm{mM}$ in $0.1 \%$ phosphoric acid.
Table 1. Linear regression, $L O D$, and $L O Q$ of the validated RPHPLC method for determination of cysteamine and cystamine

\begin{tabular}{lcc}
\hline \multicolumn{1}{c}{ Parameters } & Cysteamine & Cystamine \\
\hline Regression equation & $\mathrm{y}=0.0300 \mathrm{x}-0.0216$ & $\mathrm{y}=0.0229 \mathrm{x}-0.0882$ \\
$r^{2}$ & 0.9999 & 0.9999 \\
LOD $(\mu \mathrm{g} / \mathrm{mL})$ & 0.32 & 1.63 \\
LOQ $(\mu \mathrm{g} / \mathrm{mL})$ & 0.97 & 4.95 \\
\hline
\end{tabular}

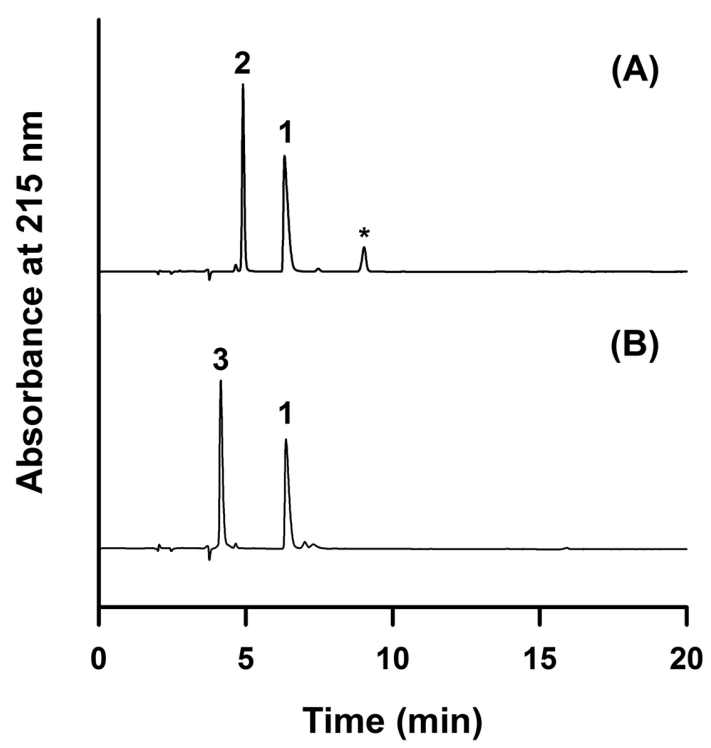

Fig. 2. Specificity of RP-HPLC method. Chromatograms of cysteamine mixed with thioglycolic acid (A) and cysteine (B). Peak 1: cysteamine, peak 2: thioglycolic acid, peak 3: cysteine, and asterisk: dimer of thioglycolic acid (dithiodiglycolic acid).

Method validation. The RP-HPLC method developed in this study was validated for specificity, linearity, LOD, LOQ, precision, accuracy, and recovery (Table 1, 2). Specificity of cysteamine was evaluated using solutions containing cysteamine with thioglycolic acid and cysteine. Thioglycolic acid and cysteine are representative reducing agents commonly used in hair cosmetics. As shown in Fig. 2, thioglycolic acid (retention time $(\mathrm{RT})=4.9 \mathrm{~min}$ ) and cysteine $(\mathrm{RT}=4.2 \mathrm{~min})$ were completely resolved

Table 2. Precision and accuracy of the validated RP-HPLC method

\begin{tabular}{|c|c|c|c|c|c|}
\hline \multirow{2}{*}{ Analyte } & \multirow{2}{*}{$\begin{array}{l}\text { Concentration } \\
(\mu \mathrm{g} / \mathrm{mL})\end{array}$} & \multicolumn{2}{|c|}{ Intra-day $(\mathrm{n}=5)$} & \multicolumn{2}{|c|}{ Inter-day $(\mathrm{n}=3)$} \\
\hline & & Precision (\%) & Accuracy (\%) & Precision (\%) & Accuracy (\%) \\
\hline \multirow{4}{*}{ Cysteamine } & 2 & 2.33 & $107.2 \pm 0.1$ & 2.71 & $104.0 \pm 0.1$ \\
\hline & 15 & 2.70 & $100.6 \pm 0.4$ & 0.35 & $100.2 \pm 0.1$ \\
\hline & 150 & 0.37 & $101.5 \pm 0.6$ & 0.49 & $101.7 \pm 0.8$ \\
\hline & 500 & 0.26 & $101.2 \pm 1.3$ & 0.68 & $100.4 \pm 3.4$ \\
\hline \multirow{4}{*}{ Cystamine } & 5 & 4.11 & $97.6 \pm 0.2$ & 3.95 & $101.8 \pm 0.2$ \\
\hline & 15 & 4.79 & $102.0 \pm 0.7$ & 0.93 & $100.9 \pm 0.1$ \\
\hline & 150 & 0.75 & $99.0 \pm 1.1$ & 1.14 & $99.7 \pm 1.7$ \\
\hline & 500 & 0.14 & $100.0 \pm 0.7$ & 0.36 & $99.9 \pm 1.8$ \\
\hline
\end{tabular}


from cysteamine $(\mathrm{RT}=6.9 \mathrm{~min})$. Linear regression analysis of cysteamine and cystamine was performed by plotting peak area $(\mathrm{y})$ versus concentration of standard solutions (x) across the range of $2-500 \mu \mathrm{g} / \mathrm{mL}$ for cysteamine and 5$500 \mu \mathrm{g} / \mathrm{mL}$ for cystamine. Calibration curves were constructed using eight points for cysteamine and seven points for cystamine. The coefficients of determination $\left(r^{2}\right)$ for cysteamine and cystamine were greater than 0.999, demonstrating excellent linearity across this concentration range (Table 1). The LOD and LOQ for cysteamine and cystamine were calculated using the slopes of the calibration curves and the standard deviations of the minimum detectable levels $(n=7)$. The LOD values for cysteamine and cystamine were 0.032 and $1.63 \mu \mathrm{g} / \mathrm{mL}$, respectively, and the LOQ values were 0.97 and $4.95 \mu \mathrm{g} / \mathrm{mL}$ for cysteamine and cystamine, respectively. Intra- and inter-day precision and accuracy were determined by quantifying standard samples at each concentration across the ranges of $2-500 \mu \mathrm{g} / \mathrm{mL}$ for cysteamine and $5-500 \mu \mathrm{g} / \mathrm{mL}$ for cystamine against standard curves prepared on each day (Table 2). The intra-day precision results for cysteamine and cystamine were $0.26-2.70 \%$ and $0.14-4.79 \%$, respectively. The inter-day precision results for cysteamine and cystamine were $0.35-2.71 \%$ and $0.36-3.95 \%$, respectively. Accuracy was defined as the mean percentage of analyte recovered in the assay (19). The intra-day accuracies for cysteamine and cystamine were 100.6-107.2\% and 97.6$102.0 \%$, respectively. The inter-day accuracies for cys-

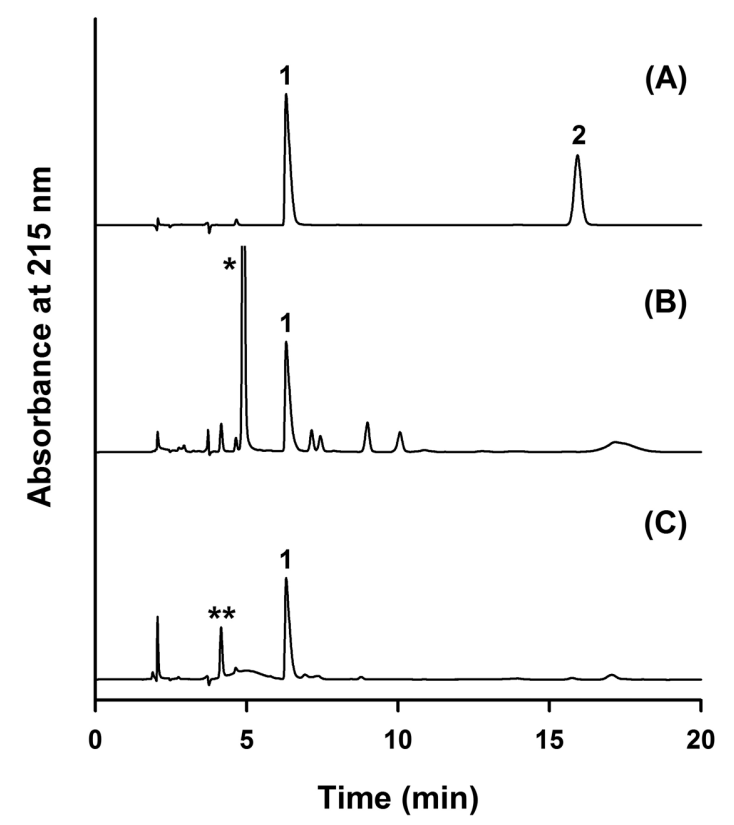

Fig. 3. Application of RP-HPLC method for the determination of cysteamine in commercial products. Chromatograms of a mixture of cysteamine and cystamine (A), product 1 (B), and product 2 (C). Peak 1: cysteamine, peak 2: cystamine, *thioglycolic acid, and ${ }^{* *}$ cysteine. teamine and cystamine were 100.2-104.0\% and 99.7-101.8\%, respectively. Recovery was assessed by comparing peak areas of cysteamine and cystamine spiked into blank cream before and after extraction. Cysteamine and cystamine were successfully analyzed without any chromatographic interference. During the extraction and analysis process, the samples were stable. The extraction recoveries for cysteamine and cystamine at $100 \mu \mathrm{g} / \mathrm{mL}$ were $100.2-102.7 \%$ and $90.6-98.7 \%$, respectively.

Analysis of cosmetic products. Two commercial cosmetic products containing cysteamine were tested to evaluate the validated RP-HPLC method (Fig. 3). The products were extracted using chloroform and water. On labeling, one product (\#1) contains cysteamine and thioglycolic acid, and the other product (\#2) contains cysteamine and cysteine as major ingredients. In both products, cysteamine was observed but its dimer impurity cystamine was not detected. Product \#1 and \#2 contained 3.6\% and 1.7\% cysteamine, respectively.

\section{DISCUSSION}

A simple RP-HPLC method for simultaneous determination of cysteamine and its dimer cystamine was successfully developed and validated. The method used a mobile phase containing $\mathrm{NaHpSO}$ as an ion-pairing agent to improve retention of cysteamine and cystamine. Since cysteamine and cystamine are cations, anionic $\mathrm{NaHpSO}$ formed an electrically neutral ion-pair with each of these analytes. These ionic interactions facilitated increased retention due to charge neutralization and the addition of a heptyl moiety to each analyte, increasing effective hydrophobicity and aiding better hydrophobic selectivity. Thioglycolic acid and cysteine are the most commonly used reducing agents in cosmetics. They are often included in formulations that contain cysteamine. Therefore, resolution of cysteamine from thioglycolic acid and cysteine is important for any analytical method used to evaluate cysteamine in cosmetic products. In the developed RP-HPLC method, thioglycolic acid and cysteine were well resolved from cysteamine and thus it allowed the contents of cysteamine to be successfully measured in the commercial products.

Although cysteamine is typically more expensive than other reducing agents such as thioglycolic acid and cysteine, cysteamine has the following advantages as a cosmetic ingredient: 1) reduction can occur at a more neutral $\mathrm{pH}$ and 2) less hair damage occurs compared with conventional reducing agents (20). Cases of cysteamine-induced allergic contact dermatitis are rare, but some patch test studies demonstrated positive results $(7,8)$. Among 1,347 hairdressers patch-tested with $0.5 \%$ cysteamine, 16 clinically relevant positive reactions $(1.2 \%)$ were observed from 
1994 to 2004. Recently, allergic contact dermatitis was assessed in 17 hairdressers in Japan, and 7 of these individuals had allergic reactions to cysteamine (8). Therefore, cysteamine may need to be included in a test series with hair waving and dyeing agents. The RP-HPLC method developed in this study has excellent potential for quality control and monitoring for risk assessments of cysteamine in cosmetics.

\section{ACKNOWLEDGMENTS}

This research was supported by a grant (14172MFDS975) from Ministry of Food and Drug Safety in 2014.

\section{CONFLICT OF INTEREST}

The authors declare that they have no conflicts of interest.

Received February 21, 2019; Revised March 8, 2019; Accepted March 10, 2019

\section{REFERENCES}

1. Coloso, R.M., Hirschberger, L.L., Dominy, J.E., Lee, J.I. and Stipanuk, M.H. (2006) Cysteamine dioxygenase: evidence for the physiological conversion of cysteamine to hypotaurine in rat and mouse tissues. Adv. Exp. Med. Biol., 583, 2536.

2. Besouw, M., Masereeuw, R., van den Heuvel, L. and Levtchenko, E. (2013) Cysteamine: an old drug with new potential. Drug Discov. Today, 18, 785-792.

3. Charrier, C., Rodger, C., Robertson, J., Kowalczuk, A., Shand, N., Fraser-Pitt, D., Mercer, D. and O'Neil, D. (2014) Cysteamine (Lynovex(R)), a novel mucoactive antimicrobial \& antibiofilm agent for the treatment of cystic fibrosis. Orphanet J. Rare Dis., 9, 189.

4. Medic, G., van der Weijden, M., Karabis, A. and Hemels, M. (2017) A systematic literature review of cysteamine bitartrate in the treatment of nephropathic cystinosis. Curr. Med. Res. Opin., 33, 2065-2076.

5. Marks, J.G., Jr., Belsito, D.V., DeLeo, V.A., Fowler, J.F., Jr., Fransway, A.F., Maibach, H.I., Mathias, C.G., Pratt, M.D., Rietschel, R.L., Sherertz, E.F., Storrs, F.J. and Taylor, J.S. (2000) North American Contact Dermatitis Group patch-test results, 1996-1998. Arch. Dermatol., 136, 272-273.

6. Landers, M.C., Law, S. and Storrs, F.J. (2003) Permanentwave dermatitis: contact allergy to cysteamine hydrochloride. Am. J. Contact Dermat., 14, 157-160.

7. Uter, W., Bensefa-Colas, L., Frosch, P., Gimenez-Arnau, A., John, S.M., Lepoittevin, J.P., Liden, C., White, I.R. and Duus Johansen, J. (2015) Patch testing with hair cosmetic series in Europe: a critical review and recommendation. Contact Derm., 73, 69-81.

8. Nishioka, K., Koizumi, A. and Takita, Y. (2019) Allergic contact dermatitis caused by cysteamine hydrochloride in permanent wave agent-A new allergen for hairdressers in Japan. Contact Derm., 80, 174-175.

9. Kusmierek, K., Chwatko, G., Glowacki, R. and Bald, E. (2009) Determination of endogenous thiols and thiol drugs in urine by HPLC with ultraviolet detection. J. Chromatogr. B Analyt. Technol. Biomed. Life Sci., 877, 3300-3308.

10. Garcia, A.J. and Apitz-Castro, R. (2002) Plasma total homocysteine quantification: an improvement of the classical high-performance liquid chromatographic method with fluorescence detection of the thiol-SBD derivatives. J. Chromatogr. B Analyt. Technol. Biomed. Life Sci., 779, 359-363.

11. Ichinose, S., Nakamura, M., Maeda, M., Ikeda, R., Wada, M., Nakazato, M., Ohba, Y., Takamura, N., Maeda, T., Aoyagi, K. and Nakashima, K. (2009) A validated HPLC-fluorescence method with a semi-micro column for routine determination of homocysteine, cysteine and cysteamine, and the relation between the thiol derivatives in normal human plasma. Biomed. Chromatogr., 23, 935-939.

12. Kusmierek, K. and Bald, E. (2008) Measurement of reduced and total mercaptamine in urine using liquid chromatography with ultraviolet detection. Biomed. Chromatogr., 22, 441-445.

13. Kusmierek, K., Chwatko, G., Glowacki, R., Kubalczyk, P. and Bald, E. (2011) Ultraviolet derivatization of low-molecular-mass thiols for high performance liquid chromatography and capillary electrophoresis analysis. J. Chromatogr. $B$ Analyt. Technol. Biomed. Life Sci., 879, 1290-1307.

14. Kataoka, H., Imamura, Y., Tanaka, H. and Makita, M. (1993) Determination of cysteamine and cystamine by gas chromatography with flame photometric detection. J. Pharm. Biomed. Anal., 11, 963-969.

15. Kataoka, H., Tanaka, H. and Makita, M. (1994) Determination of total cysteamine in urine and plasma samples by gas chromatography with flame photometric detection. J. Chromatogr. B, Biomed. Appl., 657, 9-13.

16. ICH (2005) ICH Harmonised Tripartite Guideline Q2(R1): Validationof Analytical Procedures, ICH, Geneva.

17. Park, E.J., Na, D.H., Shin, Y.H. and Lee, K.C. (2008) Liquid chromatography-mass spectrometric method for the sensitive determination of niflumic acid in human plasma and its application to pharmacokinetic study of talniflumate tablet. J. Chromatogr. B Analyt. Technol. Biomed. Life Sci., 876, 159-162.

18. Yoon, J., Kim, Y., Kim, K.B., Park, E.J. and Na, D.H. (2018) Reversed-phase high-performance liquid chromatographic method for the determination of Permaton Red (D\&C Red No. 36) in cosmetics. Bull. Korean Chem. Soc., 39, 12191222.

19. Park, E.J., Kim, M.S., Choi, Y.L., Shin, Y.H., Lee, H.S. and Na, D.H. (2012) Liquid chromatography-tandem mass spectrometry to determine the stability of collagen pentapeptide (KTTKS) in rat skin. J. Chromatogr. B Analyt. Technol. Biomed. Life Sci., 905, 113-117.

20. Isaksson, M. and van der Walle, H. (2007) Occupational contact allergy to cysteamine hydrochloride in permanentwave solutions. Contact Derm., 56, 295-296. 